\title{
Multi-species simulation of porous sand and water mixtures
}

\author{
ANDRE PRADHANA TAMPUBOLON, University of California, Los Angeles \\ THEODORE GAST, University of California, Los Angeles and Jixie Effects \\ GERGELY KLÁR, DreamWorks Animation \\ CHUYUAN FU, University of California, Los Angeles \\ JOSEPH TERAN, University of California, Los Angeles and Jixie Effects \\ CHENFANFU JIANG, University of Pennsylvania and Jixie Effects \\ KEN MUSETH, DreamWorks Animation
}

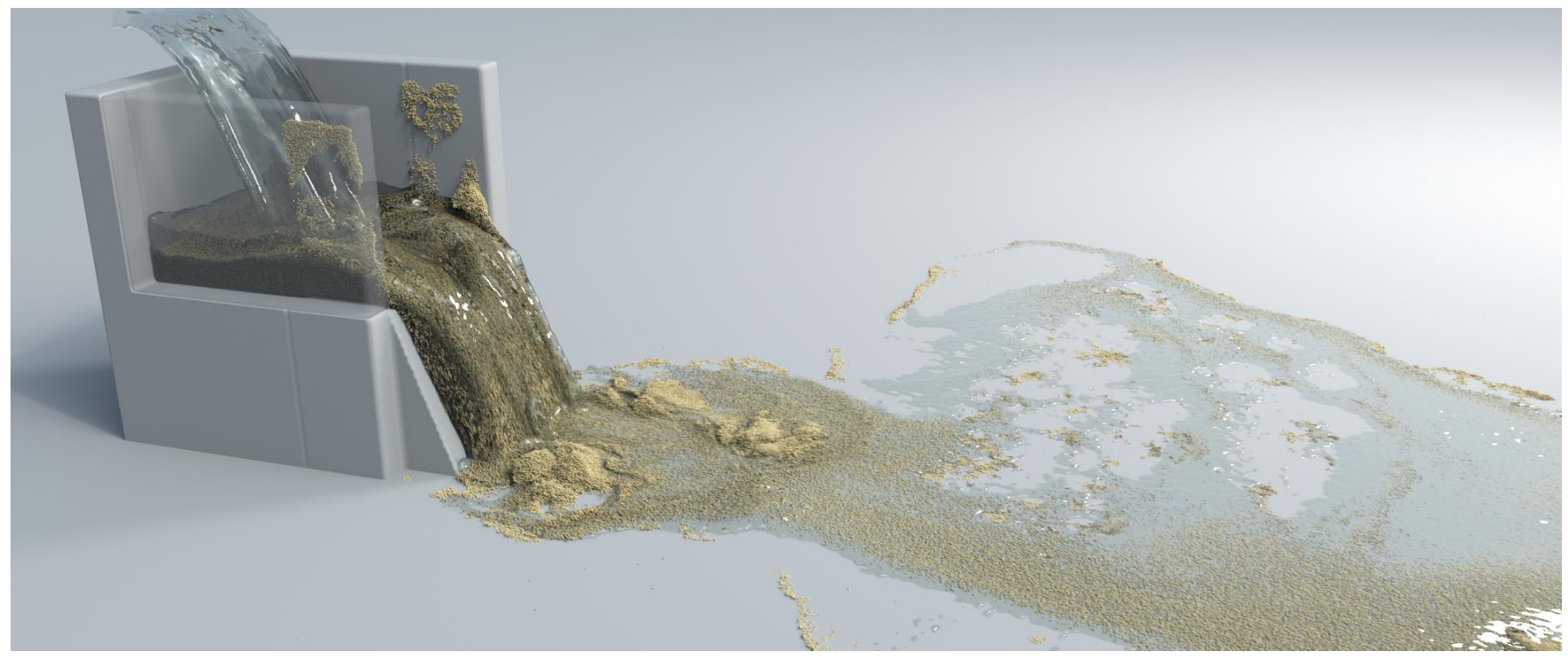

Fig. 1. Dam breach. Water pours in from a reservoir and slowly erodes a dam. As water seeps into the sand, its cohesivity decreases. When it eventually breaks, the landslide creates interesting dynamics in the debris flow.

We present a multi-species model for the simulation of gravity driven landslides and debris flows with porous sand and water interactions. We use continuum mixture theory to describe individual phases where each species individually obeys conservation of mass and momentum and they are coupled through a momentum exchange term. Water is modeled as a weakly compressible fluid and sand is modeled with an elastoplastic law whose cohesion varies with water saturation. We use a two-grid Material Point Method to discretize the governing equations. The momentum exchange term in the mixture theory is relatively stiff and we use semi-implicit time stepping to avoid associated small time steps. Our semi-implicit treatment is explicit in plasticity and preserves symmetry of force linearizations. We develop a novel regularization of the elastic part of the sand constitutive model that better mimics plasticity during the implicit solve to prevent numerical cohesion artifacts that would otherwise have occurred. Lastly, we develop

Permission to make digital or hard copies of all or part of this work for personal or classroom use is granted without fee provided that copies are not made or distributed for profit or commercial advantage and that copies bear this notice and the full citation on the first page. Copyrights for components of this work owned by others than the author(s) must be honored. Abstracting with credit is permitted. To copy otherwise, or republish, to post on servers or to redistribute to lists, requires prior specific permission and/or a fee. Request permissions from permissions@acm.org.

(c) 2017 Copyright held by the owner/author(s). Publication rights licensed to ACM. 0730-0301/2017/7-ART105 \$15.00

DOI: http://dx.doi.org/10.1145/3072959.3073651 an improved return mapping for sand plasticity that prevents volume gain artifacts in the traditional Drucker-Prager model.

CCS Concepts: • Computing methodologies $\rightarrow$ Physical simulation;

Additional Key Words and Phrases: MPM, elastoplasticity, porous media

ACM Reference format:

Andre Pradhana Tampubolon, Theodore Gast, Gergely Klár, Chuyuan Fu, Joseph Teran, Chenfanfu Jiang, and Ken Museth. 2017. Multi-species simulation of porous sand and water mixtures. ACM Trans. Graph. 36, 4, Article 105 (July 2017), 11 pages.

DOI: http://dx.doi.org/10.1145/3072959.3073651

\section{INTRODUCTION}

While wet sand is both ubiquitous and literally child's play, simulating the underlying interaction of water and sand certainly is not. In fact, this type of visual effect is rarely seen in feature movie productions, typically because it is considered too complex and difficult to achieve with existing particle-based simulation techniques. This is surprising given that water simulations, and to a lesser extent also sand simulations, are routinely undertaken in VFX. It is the complex nature of the changing material behaviors resulting from 


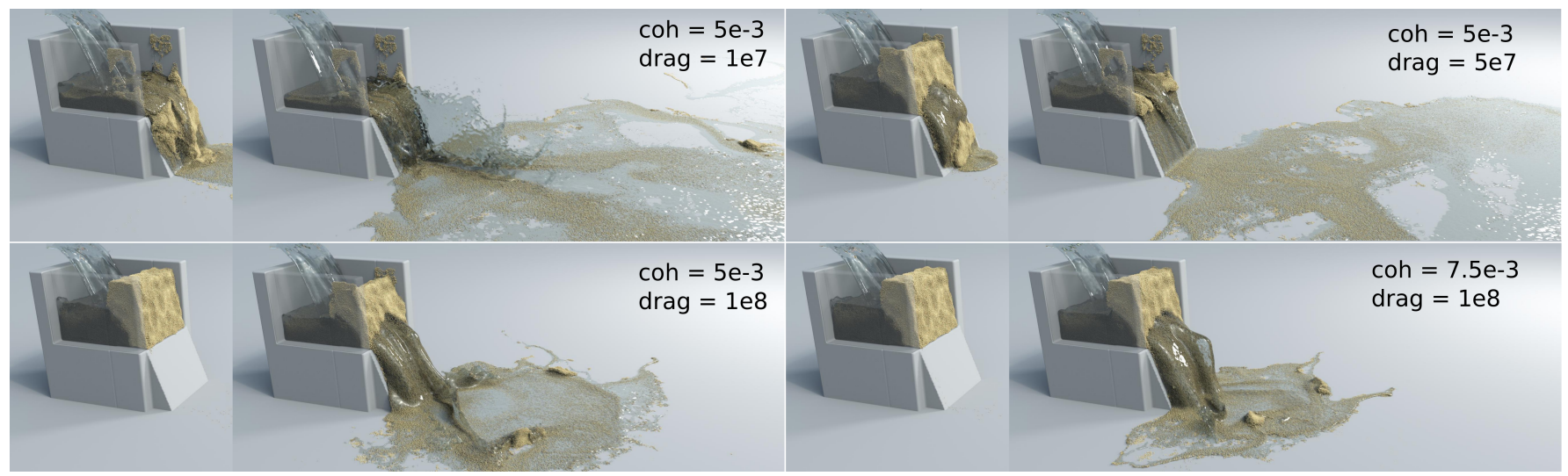

Fig. 2. When the levee breaks. Here we demonstrate the effects of our parameters on levee wall integrity. A simulation with lower drag momentum exchange coefficient and lower cohesion will fail more easily.

the mixing of sand and water that complicates simulation. However, we argue that this is precisely what makes the animation of wet sand so visually intriguing and hence desirable to master.

Most practitioners in the VFX industry would agree that animation of water is a mature area of research, as evident by its abundance and the fact that several excellent commercial solutions exist today, c.f. Houdini, Maya, and RealFlow just to mention a few. Conversely, animation of sand is considered challenging by most, and is still a subject of significant research. Although the Material Point Method (MPM) [Sulsky et al. 1995] has been demonstrated to produce encouraging results for sand simulations, commercial solutions tend to use simpler particle based techniques, like Position Based Dynamics (PBD), which is easier to implement, but less well founded in continuum theory. However, mixtures of water and sand present a completely new set of simulation challenges for which, to the best of our knowledge, there is still no established best practice in VFX, let alone off-the-shelf commercial solutions. While there are many successful examples of wet sand animation, they tend to be based on art-directed constrained simulations or even procedural particle systems. Given the success of simulating sand with MPM it is natural to explore a similar approach for wet sand. Such a unified MPM description of the mixing of water and sand is exactly what motivated our work.

In this paper, we develop a method for multi-species MPM to allow interactions between sand and water for gravity driven landslides and debris flows. We resolve wet sand transitions from cohesive rigid grains to flowing slurries as water saturation increases. We use a semi-implicit time stepping scheme to deal with stiff terms in the multi-species momentum exchange. The semi-implicit approach in [Stomakhin et al. 2013] produces artificial cohesive effects for sand simulations. We introduce a unilateral modification to the elastic energy function that better mimics the effect of plasticity to prevent these cohesive artifacts. Furthermore, we develop an improved return mapping for Drucker-Prager plasticity that reduces the volume gain observed with lower grid resolutions. In our multi-species treatment, we use the two grid scheme proposed by Bandara and Soga [2015]. Each material point is associated with a set of grid nodes and the interaction between the two species is via an interaction term proportionate to relative velocity between the phases. This term is stiff for practical porosities, and it is impractical to do explicit simulation since it would require a time-step restriction on the order of $\Delta t \approx 10^{-6}-10^{-5}$. Our semi-implicit treatment allows us to use a time step on the order of $\Delta t=10^{-3}$. Furthermore, using our modification to the elastic energy function and lagged plasticity approach, the system to be solved remains symmetric. We summarize our contributions as:

- A unilateral elastic constitutive model modification framework that removes numerical cohesion with semi-implicit time stepping and plasticity.

- An improved return mapping algorithm for Drucker-Prager plasticity with reduced volume gain artifacts.

- A semi-implicit two grid MPM discretization of stiff interaction terms in multi-species continuum equations.

- A model for wet sand cohesion based on water saturation.

\section{PREVIOUS WORK}

Probably the earliest work on water and sand in computer graphics is by Peachey [1986]. Rungjiratananon et al. simulate sand-water interaction in real-time using a hybrid Smoothed Particles Hydrodynamics (SPH) and Discrete-Element Method (DEM) approach [Rungjiratananon et al. 2008]. Lenaerts and Dutre [2009] also couple water with porous granular materials using SPH. Notably, these approaches capture a wider range of porous phenomena than that considered in our approach. While we focus on gravity driven landslides and debris flows, their approaches more accurately capture surface tension driven effects like capillary action drawing water into dry sand. They can also handle landslides and debris flows, but they do so with SPH, whereas we develop an MPM approach that naturally allows for implicit time stepping and high resolution simulation. Other graphics approaches have shown the efficacy of hybrid Lagrangian/Eulerian approaches like FLIP and MPM, including sand [Daviet and Bertails-Descoubes 2016; Klár et al. 2016; Narain et al. 2010; Zhu and Bridson 2005] and various other elastoplastic materials [Jiang et al. 2015; Ram et al. 2015; Stomakhin et al. 2013, 2014; 

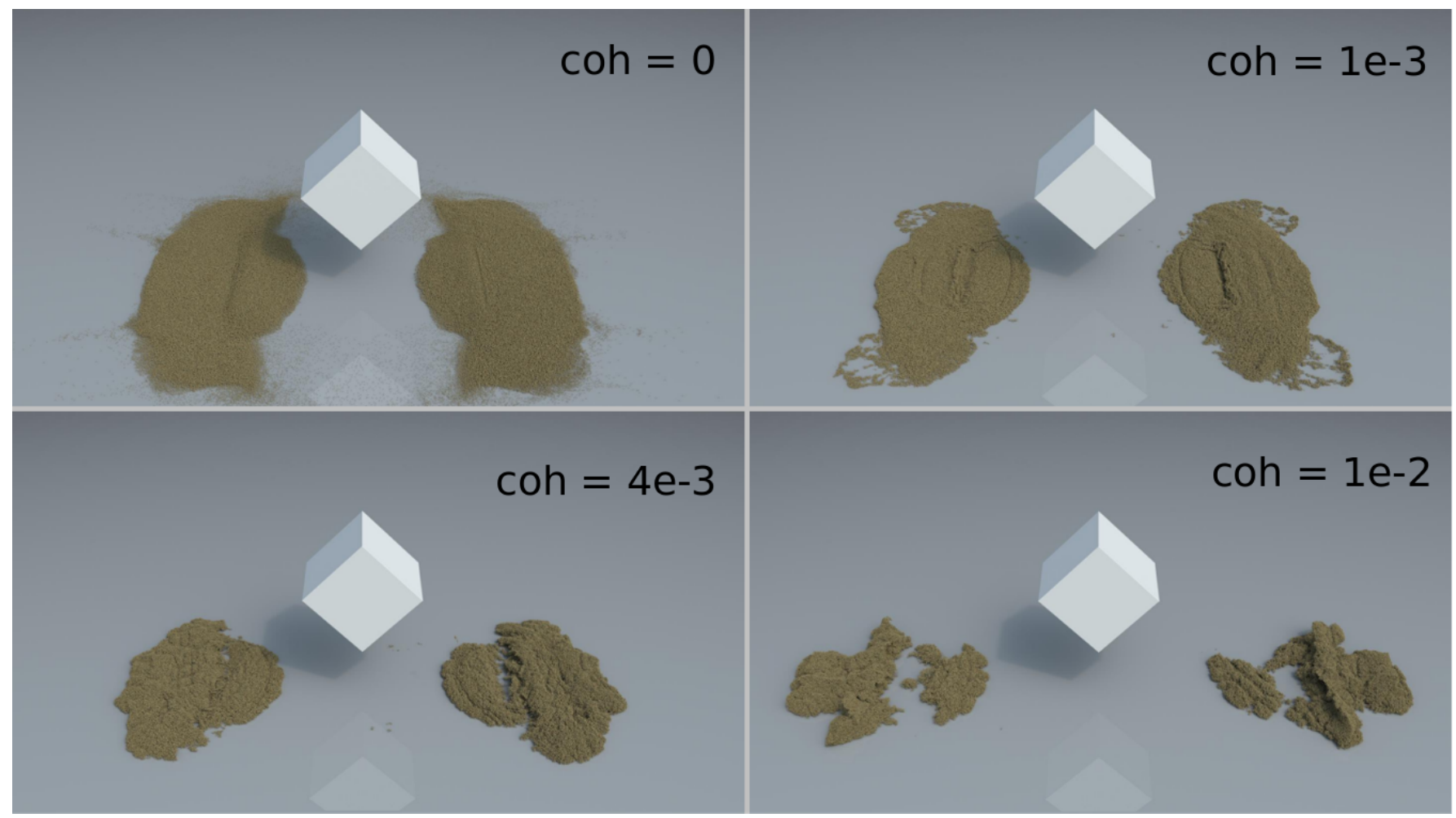

Fig. 3. Dropping sand on wedges. A box of sand with various values of cohesion is dropped onto a wedge. The top left simulation is done using an explicit time-stepping scheme, while all the others are done using a semi-implicit time-stepping scheme.

Yue et al. 2015]. Unilateral incompressibility is an effective assumption for granular materials [Alduán and Otaduy 2011; Daviet and Bertails-Descoubes 2016; Ihmsen et al. 2013; Narain et al. 2010].

Our approach is the first MPM technique in graphics that considers multi-species modeling for porous sand/water. However, mixture theory and multi-species simulations have been used for a wide range of effects in computer graphics. Nielsen and Osterby [2013] simulate spray and mist with a two-continua mixture model. Takashi et al. [2003] use the Cubic Interpolation Propagation method to couple spray, water and foam continua. Losasso et al. [2008] and Yang et al. [2014] also represent spray and dense water with multiple phases. Similar multi-species interaction ideas have been used for bubbles in incompressible flow [Mihalef et al. 2009; Ren et al. 2015; Song et al. 2005; Thürey et al. 2007]. Liu et al. [2008] use two continua to simulate mixtures of air and dust. Multi-species approaches have been used for miscible and immiscible fluids [Bao et al. 2010; He et al. 2015; Kang et al. 2010; Ren et al. 2014; Yang et al. 2015].

Various researchers in engineering have shown the efficacy of simulating water and soil interactions with the MPM. Abe et al. [2014] solve coupled hydromechanical problems of fluid-saturated soil subjected to large deformation with a two grid MPM algorithm based on Biot's mixture theory. Bandara et al. use a single grid MPM method for saturated and unsaturated soils that undergo large deformations in [Bandara et al. 2016] and two grid MPM to represent soil skeleton and pore water layers in [Bandara and Soga 2015]. Jassim et al. [2013] also develop a two grid MPM approach for soil mechanics problems.
Mast et al. [Mast et al. 2014] use MPM to simulate large deformation, gravity-driven landslides of porous soil. Mackenzie-Helnwein et al. [2010] examine the multi-species momentum exchange terms for problems with liquefaction, landslides, and sedimentation with two grid MPM.

\section{MATHEMATICAL BACKGROUND}

We model sand and water as a multi-species continuum using mixture theory [Atkin and Craine 1976; Borja 2006]. With this approach, each species is given distinct material properties, and their motion is derived from distinct velocity fields. This kinematic assumption allows sand and water to occupy the same points in space at the same time to create the mixture. We use superscript $s$ to represent sand quantities and superscript $w$ to represent water quantities. With this convention, the primary state is defined in terms of mass density $\rho^{\alpha}(\mathbf{x}, t)$ and material velocity $\mathbf{v}^{\alpha}(\mathbf{x}, t)$, where $\alpha=s, w$. The momentum density of phase $\alpha$ is given by $\rho^{\alpha} \mathbf{v}^{\alpha}$. The total mass density of the mixture is the sum $\rho=\rho^{s}+\rho^{w}$ and total momentum is the sum $\rho \mathbf{v}=\rho^{s} \mathbf{v}^{s}+\rho^{w} \mathbf{v}^{w}$. This defines the velocity $\mathbf{v}=\frac{1}{\rho}\left(\rho^{s} \mathbf{v}^{s}+\rho^{w} \mathbf{v}^{w}\right)$ of the mixture as the mass-averaged velocity of the constituents.

Each species obeys the following conservation of mass with respect to its own motion

$$
\frac{D^{\alpha} \rho^{\alpha}}{D t}+\rho^{\alpha} \nabla \cdot \mathbf{v}^{\alpha}=0
$$



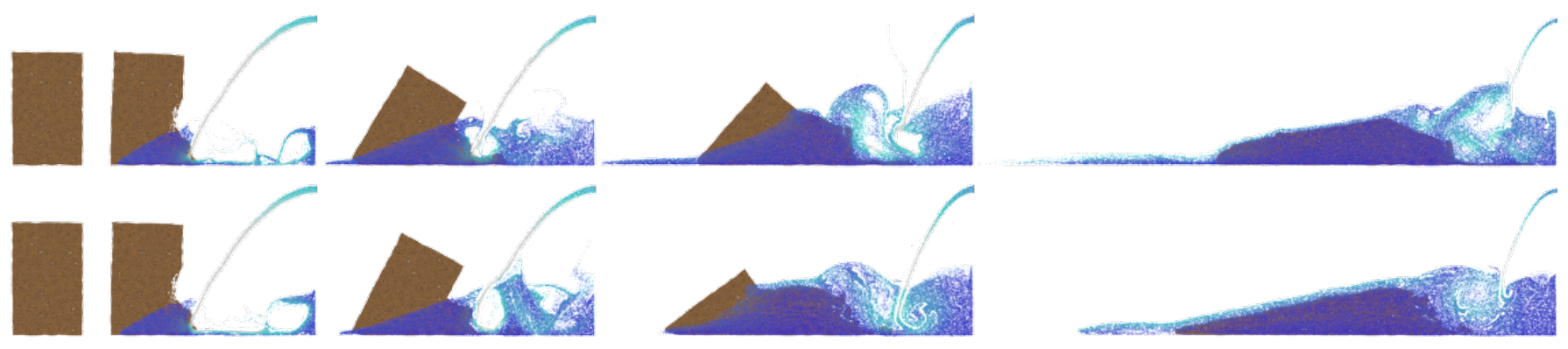

Fig. 4. 2D dam breach. A 2D dam breach simulation showing the effect of reversible term from Bandara and Soga [Bandara et al. 2016]. The figure depicts the beginning, middle, and the end of a dam breach scenario. At the top is a simulation with no reversible term (as in [Mackenzie-Helnwein et al. 2010]) and at the bottom is a simulation with the reversible term.

Here the operator $\frac{D^{\alpha} f}{D t}=\frac{\partial f}{\partial t}+\mathbf{v}^{\alpha} \cdot \nabla f$ is the material (or total) derivative of function $f$ with respect to the motion of either the sand ( $\alpha=s$ ) or water $(\alpha=w)$ phases. By summing over $\alpha$ in Equation 1 , we obtain the standard conservation of mass

$$
\frac{D \rho}{D t}+\rho \nabla \cdot \mathbf{v}=0
$$

by noting that $\rho \frac{D f}{D t}=\sum_{\alpha} \rho^{\alpha} \frac{D^{\alpha} f}{D t}$ where $\frac{D f}{D t}=\frac{\partial f}{\partial t}+\mathbf{v} \cdot \nabla f$ is the material derivative of function $f$ with respect to the motion of the mixture. Each species also obeys conservation of linear momentum as

$$
\rho^{\alpha} \frac{D^{\alpha} \mathbf{v}^{\alpha}}{D t}=\nabla \cdot \sigma^{\alpha}+\mathbf{p}^{\alpha}+\rho^{\alpha} \mathbf{g},
$$

where $\mathbf{p}^{\alpha}$ represents the transfer of momentum due to the relative motion of the constituents, $\sigma^{\alpha}$ is the partial stress tensor associated with species $\alpha$, and $\mathrm{g}$ is the gravitational acceleration. Because $\mathbf{p}^{\alpha}$ represent the exchange of momenta between species, the sum $\sum_{\alpha} \mathbf{p}^{\alpha}=\mathbf{0}$ must be zero to not affect the total linear momentum of the mixture. Indeed with this constraint we can show by summing over $\alpha$ in Equation 3

$$
\rho \frac{D \mathbf{v}}{D t}=\nabla \cdot \sigma+\rho \mathbf{g}
$$

where we have summed over $\alpha$ and introduced $\sigma=\sum_{\alpha} \sigma^{\alpha}$, which denotes the Cauchy stress in the mixture expressed as the sum of the partial stresses in each species. In other words, with this notion of the Cauchy stress, conservation of linear momentum for the individual species implies conservation of linear momentum for the mixture.

\subsection{Sand elastoplasticity}

We define the constitutive behavior of sand with elastoplasticity as in Klár et al. [2016]. However, their approach was for dry sand. Wet sand is capable of retaining its shape via tensile stresses and the unmodified Drucker-Prager yield condition does not admit this. We modify the model slightly to include cohesive stresses. The amount of cohesion varies with the saturation level of water in the mixture. We assume that the sand partial stress $\sigma^{s}$ is defined in terms of the hyperelastic potential energy density $\psi^{s}$ as

$$
\boldsymbol{\sigma}^{s}=\frac{1}{\operatorname{det}\left(\mathbf{F}^{s}\right)} \frac{\partial \psi^{s}}{\partial \mathbf{F}}\left(\mathbf{F}^{s, E}\right) \mathbf{F}^{s, E^{\top}}
$$

Here, $\mathbf{F}^{s}$ is the deformation gradient of the sand motion, which evolves as $\frac{D^{s}}{D t} \mathbf{F}^{s}=\nabla \mathbf{v}^{s} \mathbf{F}^{s}$. The appearance of the $\frac{1}{\operatorname{det}\left(\mathrm{F}^{s}\right)}$ and $\mathbf{F}^{s, E^{\top}}$ terms arise because we write the potential energy density in terms of the deformation gradient. With this convention, its derivative gives rise to the first Piola-Kirchhoff stress and these terms are needed to transform it into the Cauchy stress. As typically done in finite strain elastoplasticity [Bonet and Wood 2008], it is decomposed into $\mathbf{F}^{s}=\mathbf{F}^{s, E} \mathbf{F}^{s, P}$ to define a plastic flow. For sand, $\mathbf{F}^{s, E}$ represents the remembered compression and shearing, while $\mathrm{F}^{s, P}$ represents the forgotten sliding and separation. We use the Drucker-Prager [Drucker and Prager 1952] plastic flow and yield condition to determine the evolution of the elastic $\left(\mathbf{F}^{s, E}\right)$ and plastic $\left(\mathbf{F}^{s, P}\right)$ parts of the deformation gradient. The Drucker-Prager yield condition is defined from the constraint that the shear stress should be no larger than the compressive normal stress in all directions. This expresses a mechanical interaction that is consistent with Coulomb friction. While dry sand is modeled effectively with this assumption, it precludes the effects of cohesion. However, cohesive effects can be modeled by modifying the elastic stress yield condition to be

$$
c_{F} \operatorname{tr}\left(\sigma^{s}\right)+\left\|\sigma^{s}-\frac{\operatorname{tr}\left(\sigma^{s}\right)}{d} \mathbf{I}\right\|_{F} \leq c_{C},
$$

where $d=2,3$ is the spatial dimension, $c_{C} \geq 0$ increases with the amount of cohesion in the material and $c_{F} \geq 0$ increases with amount of friction between grains. E.g. for dry sand in Klár et al. [2016] they used $c_{C}=0$. A positive $c_{C}$ shifts the yield surface along the hydrostatic axis (the line where $\operatorname{tr}\left(\sigma^{s}\right)=0$ ), which allows the material to exhibit stress under tension and thus cohere to itself. Here we model cohesivity as a function of water saturation in the sand. This is naturally measured in terms of the volume fraction of water in the mixture $\phi^{w}=\frac{\rho^{w}}{\rho}$ so that $c_{C}=c_{C}\left(\phi^{w}\right)$.

Inequality 6 is referred to as the plastic yield condition, if it is satisfied, there will be no further plastic deformation. The boundary of the region in stress space defined by $c_{F} \operatorname{tr}\left(\sigma^{s}\right)+\left\|\sigma^{s}-\frac{\operatorname{tr}\left(\sigma^{s}\right)}{d} \mathbf{I}\right\|_{F}=$ $c_{C}\left(\phi^{w}\right)$ is called the yield surface. For states of stress on the yield surface, plastic flow will commence when a perfectly elastic assumption would drive the stress out of the region. The plasticity functions as a means of satisfying this inequality constraint.

3.1.1 Unilateral hyperelasticity. Here we describe the elastic part of the constitutive behavior for the sand phase. This is largely 


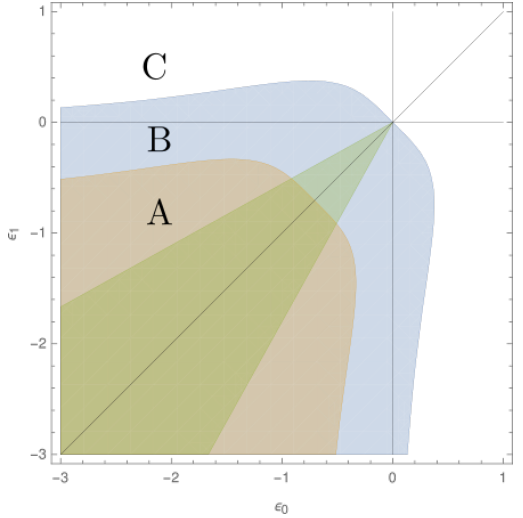

Fig. 5. Multiplier region. The contour plot of the multiplier in strain space. Region $\mathrm{A}$ is where the multiplier is equal to 1 , region $\mathrm{C}$ is where the multiplier is 0 , and region $\mathrm{B}$ is where the multiplier transitions from 1 to 0 in a $C^{2}$ manner. The green cone denotes the Drucker-Prager cone in principal strain space.

the same as in Klár et al. [2016] where the elastic potential energy density is defined in terms of the logarithmic strain $\epsilon$ as $\psi^{s}\left(\mathbf{F}^{s}\right)=\tilde{\psi}^{s}(\boldsymbol{\epsilon})=\mu \operatorname{tr}\left(\boldsymbol{\epsilon}^{2}\right)+\frac{\lambda}{2} \operatorname{tr}(\boldsymbol{\epsilon})$ where $\mathbf{F}^{s}=\mathbf{U} \Sigma \mathbf{V}$ is the singular decomposition of $\mathbf{F}^{s}$ and $\epsilon=\log (\Sigma)$. However, we provide some novel modifications that allow for more efficient implicit time integration. We note that with the plastic yield condition from Inequality 6, much of the energy landscape has no effect since it produces stresses outside of the yield surface. To mitigate this we modify the energy density such that it smoothly transitions to zero in these regions. This has no effect on the continuous behavior of the governing equations, but it improves the performance of our semi-implicit time stepping. Essentially, our modification modifies the elastic behavior outside the yield surface to better resemble the effects of plasticity.

Our approach is similar to, and indeed inspired by the unilateral approaches in [Alduán and Otaduy 2011; Daviet and BertailsDescoubes 2016; Ihmsen et al. 2013; Narain et al. 2010]. We thus refer to our modified energy as the unilateral energy function $\tilde{\psi}^{s, U}(\boldsymbol{\epsilon})$. We define this as the product of the original energy function $\tilde{\psi}^{s}(\boldsymbol{\epsilon})$ and a multiplier $h(\boldsymbol{\epsilon})$

$$
\tilde{\psi}^{s, U}(\boldsymbol{\epsilon})=\tilde{\psi}^{s}(\boldsymbol{\epsilon}) h(\boldsymbol{\epsilon}) .
$$

The multiplier makes sure that the energy density function to transition to zero in regions outside of the yield surface (as smoothly as possible). We define it to be symmetric about the hydrostatic axis in the strain space (axis of equal strain, see Figure 5) since the original energy $\tilde{\psi}^{s}$ has this property and we wish to preserve it in $\tilde{\psi}^{s, U}$. To construct it, we partition strain space into the three regions labeled $\mathrm{A}, \mathrm{B}$, and $\mathrm{C}$ in Figure 5. In region $\mathrm{A}$, the value of the multiplier is simply one and the modified constitutive model is identical to the original one. Conversely, in region $\mathrm{C}$, the multiplier is set to zero. In region $\mathrm{B}$, the multiplier transitions from one to zero in a $C^{2}$ manner The boundary of region $\mathrm{A}$ and $\mathrm{B}$ defines two envelopes that are symmetric about the hydrostatic axis. To best preserve the behavior of sand, we want region A to cover most of the region inside of the Drucker-Prager cone, illustrated as the green cone in Figure 5.

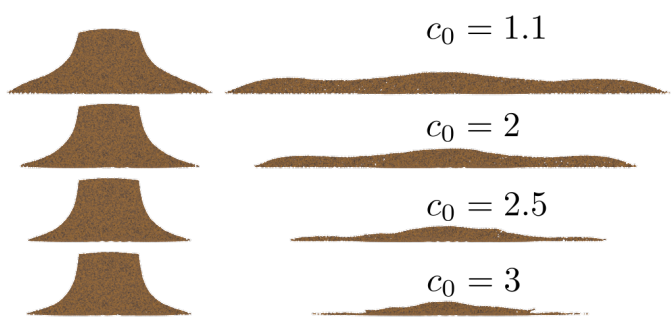

Fig. 6. Unilateral extension. We demonstrate a 2D sand column collapse with different values of $c_{0}$ coefficient in the unilateral modified energy density function. From top to bottom, $c_{0}=1.1,2,2.5,3$. The left image is of an early frame and the right is of a later frame. Here parameters $a$ and $b$ from Equation 10 are $a=-0.5$, and $b=0$. As $c_{0}$ increases, the envelopes in the unilateral extension become increasingly poor approximations of the Drucker-Prager cone and we see volume loss artifacts.

The construction of this multiplier function is done as a composition of two functions: $h_{s}$ which is a scalar function and $f$ which is a function of the strain $\epsilon$. If we let o denote the hydrostatic axis $\left(\mathbf{o}=\frac{1}{\sqrt{2}}(1,1)^{\top}\right.$ in $2 \mathrm{D}$ or $\frac{1}{\sqrt{3}}(1,1,1)^{\top}$ in $\left.3 \mathrm{D}\right)$, then we can compute $u=\boldsymbol{\epsilon} \cdot \mathbf{o}$ to be the component of the strain in the hydrostatic axis and $v=\|\boldsymbol{\epsilon}-u \mathbf{o}\|$. The function $f$ is defined as

$$
f(\epsilon)=c_{o} \frac{v^{4}}{1+|v|^{3}} .
$$

The coefficient $c_{o}$ controls the opening of the envelope of regions $\mathrm{A}$ and $\mathrm{B}$ around the hydrostatic axis. The scalar function $h_{s}$ is chosen so that the multiplier function is twice continuously differentiable, and is given by

$$
h_{s}(z)= \begin{cases}1 & \text { if } z<0 \\ 0 & \text { if } z>1 \\ 1-10 z^{3}+15 z^{4}-6 z^{5} & \text { otherwise. }\end{cases}
$$

The multiplier function is then defined for some choice of parameters $a, b$, and $s_{C}$.

$$
h(\boldsymbol{\epsilon})= \begin{cases}1 & \text { if } u+f(\boldsymbol{\epsilon})<a+s_{C} \\ 0 & \text { if } u+f(\boldsymbol{\epsilon})>b+s_{C} \\ h_{s}\left(\frac{u+f(\boldsymbol{\epsilon})-a-s_{C}}{b-a}\right) & \text { otherwise. }\end{cases}
$$

The parameter $a$ and $b$ determines the intersection of the hydrostatic axis with the boundary of region $A$ and $B$ respectively. The parameter $s_{C}$ controls a shift of this multiplier region along the hydrostatic axis.

\subsection{Water}

We model the water as nearly incompressible [Becker and Teschner 2007] with the partial stress

$$
\boldsymbol{\sigma}^{w}=-p^{w} \mathbf{I}, \quad p^{w}=k\left(\frac{1}{J^{w \gamma}}-1\right) .
$$


We note that the water pressure is related to a potential $\psi^{w}$ as $p^{w}=$ $-\frac{\partial \psi^{w}}{\partial J^{w}}\left(J^{w}\right)$, with $\psi^{w}\left(J^{w}\right)=-k\left(\frac{\left(J^{w}\right)^{(1-\gamma)}}{1-\gamma}-J^{w}\right)$. This pressure $p^{w}$ is designed to stiffly penalize the volume change of the water phase, which is characterized in terms of the determinant of the water deformation gradient $J^{w}=\operatorname{det}\left(\mathbf{F}^{w}\right)$. As with the solid phase, the fluid deformation gradient evolves according to $\frac{D^{w}}{D t} \mathrm{~F}^{w}=\nabla \mathbf{v}^{w} \mathrm{~F}^{w}$. Intuitively, $J^{w}$ is the ratio of the current to initial local volume of material in the water phase. It evolves as

$$
\frac{D^{w}}{D t} J^{w}=\nabla \cdot \mathbf{v}^{w} J^{w}
$$

Here $k$ is the bulk modulus of the water and $\gamma$ is a term that more stiffly penalizes large deviations from incompressibility.

\subsection{Momentum exchange}

The momentum exchange terms $\mathbf{p}^{s}, \mathbf{p}^{w}$ for water and porous sand interactions can generally be viewed as a combination of dissipative and reversible interactions [Borja 2006]. We follow the formulation of Bandara and Soga [2016] because, like them, we are concerned primarily with gravity driven flows such as fast catastrophic landslides and debris flows. Their formulation assumes

$$
\mathbf{p}^{s}=c_{E}\left(\mathbf{v}^{w}-\mathbf{v}^{s}\right)+p^{w} \nabla \phi^{w}, \mathbf{p}^{w}=-\mathbf{p}^{s}
$$

where $c_{E}=\frac{n^{2} \rho^{w} g}{\hat{k}}$ and $n$ is the sand porosity, $\hat{k}$ is the sand permeability and $g$ is the gravitational acceleration, $\phi^{w}=\frac{\rho^{w}}{\rho}$ is the water volume fraction and $p^{w}$ is the water pressure. The first term represents viscous forces generated by sand particles moving through the fluid. Although it can be conceived from the view of an idealized particle moving through a Stokes fluid, it simply amounts to a Coulomb-friction-like response [Mackenzie-Helnwein et al. 2010]. The second term is often called the "buoyancy term" in mixture theories [Robert and Soga 2013]. It can be conceived from entropy equilibrium constraints or from the physical consideration that the pore fluid pressure multiplying the porosity is appropriate notion of reversible pressure, however there is some debate about its appropriateness outside of immiscible mixtures [Drumheller 2000] Mackenzie-Helnwein et al. [2010] omit the second term and view the momentum exchange terms as purely dissipative processes. We also follow this approach for the majority of the examples presented in the paper, however we include one example demonstrating its effect in Figure 4. Even with the inclusion of the active term from [Bandara et al. 2016], we are only capable of simulating a rather narrow range of porous media phenomena. While this is sufficient for landslides and debris flows, phenomena such as capillary action drawing water into dry sand is not achievable in our approach.

\subsection{Cohesion and Saturation}

As in Robert and Soga in [2013], we assume that the sand cohesion varies as a function of water saturation. We measure saturation as the percentage of water in the mixture which we estimate as the ratio of the density of the water phase to the total density $\phi^{w}=\frac{\rho^{w}}{\rho^{w}+\rho^{s}}$. The cohesion of sand is zero when it is completely dry $\left(\phi^{w}=0\right)$. Intuitively, as dry sand becomes saturated with more water, the cohesivity of the wet sand should increase as wet sand tends to better

\begin{tabular}{|c|c|c|c|}
\hline Variable & Where & Species & Meaning \\
\hline $\mathrm{g}$ & - & - & gravitational constant \\
\hline$c_{E}$ & - & - & drag coefficient \\
\hline$m_{p}^{\alpha}$ & particles & both & particle mass \\
\hline$V_{p}^{\alpha 0}$ & particles & both & initial particle volume \\
\hline $\mathrm{x}_{p}^{\alpha, n}, \mathrm{x}_{p}^{\alpha, n+1}$ & particles & both & particle position \\
\hline $\mathrm{v}_{p}^{\alpha, n}, \mathrm{v}_{p}^{\alpha, n+1}$ & particles & both & particle velocity \\
\hline $\mathrm{F}_{p}^{n}, \mathrm{~F}_{p}^{n+1}$ & particles & matrix & deformation gradient \\
\hline $\mathrm{F}_{p}^{s E, n}, \mathrm{~F}_{p}^{s E, n+1}$ & particles & sand & sand elastic deformation gradient \\
\hline$\overline{\mathrm{F}_{p}^{s P, n}, \mathrm{~F}_{p}^{s P, n+1}}$ & particles & sand & plastic deformation gradient \\
\hline$J_{p}^{w, n}, J_{p}^{w, n+1}$ & particles & water & determinant deformation gradient \\
\hline$\phi_{p}^{s, n}$ & particle & sand & water saturation \\
\hline$c_{C_{p}}^{s, n}$ & particle & sand & cohesion \\
\hline$v_{c p}^{s}$ & particle & sand & volume correction scalar \\
\hline$(\nabla \mathbf{v})_{p}^{\alpha}$ & particles & matrix & grid-based velocity gradient \\
\hline$m_{i}^{\alpha, n}$ & grid & both & grid node mass \\
\hline $\mathrm{v}_{i}^{\alpha, n}$ & grid & both & rasterized velocity \\
\hline $\mathbf{v}_{i}^{\alpha, n+1}$ & grid & both & final grid velocity \\
\hline $\mathrm{x}_{i}^{\alpha, n}$ & grid & both & Cartesian grid node locations \\
\hline$\hat{\mathbf{x}}_{i}^{\alpha, n+1}$ & grid & both & grid positions moved by $\mathrm{v}_{i}^{\alpha, n+1}$ \\
\hline$\phi_{i}^{w, n+1}$ & grid & mixed & water saturation \\
\hline $\mathrm{f}_{i}^{\alpha}$ & grid & both & internal forces \\
\hline
\end{tabular}
hold its shape. Indeed this was observed in the work of Robert and
Soga in [2013]. However, they also observe that this increase only continues to a maximal value $c_{C}^{\max }$ when the saturation is around $\phi^{w}=0.4$. Beyond this point the sand becomes more compliant to flowing and less cohesively elastic. In all of our multispecies examples, we model water interaction with wet sand that is capable of holding its shape. We thus set the sand cohesion to be initially maximal, even in the absence of the water phase. Based on the observations in Robert and Soga [2013], we then assume that the cohesion decreases linearly with increasing saturation beyond this point (with cohesion equal to zero at full saturation $\phi^{w}=1$ ).

\section{DISCRETIZATION}

First, we explain the notation used in the discretization section. There are two sets of grids: one is associated with sand material and the other is associated with water. As with the continuous equations, the superscript $\alpha=s, w$ indicates the corresponding species. Whenever a symbol has a subscript $i$ or $j$, this denotes one degree of freedom in grid node index $i$ or $j$. Subscript $p$ is used to denote attributes that belong to a particle. A symbol that is not followed by a subscript refers to the whole collection of grid nodes as degrees of freedom. For example $\mathbf{v}^{s, n+1}$ refers to all of the sand grid nodes that are active at step $n+1$.

We discretize the continuum equation using the Material Point Method (MPM). As in other approaches in the engineering literature [Bandara and Soga 2015; Jassim et al. 2013; Mackenzie-Helnwein et al. 2010], we use two sets of grids (Figure 7): one for the solid particles and the other for the water particles. This is the primary difference between our approach and others recently used in the graphics literature. The overview of the algorithm is as follows:

(1) Transfer to grids: Transfer the mass and momentum of each species to its corresponding grid (§4.1). 

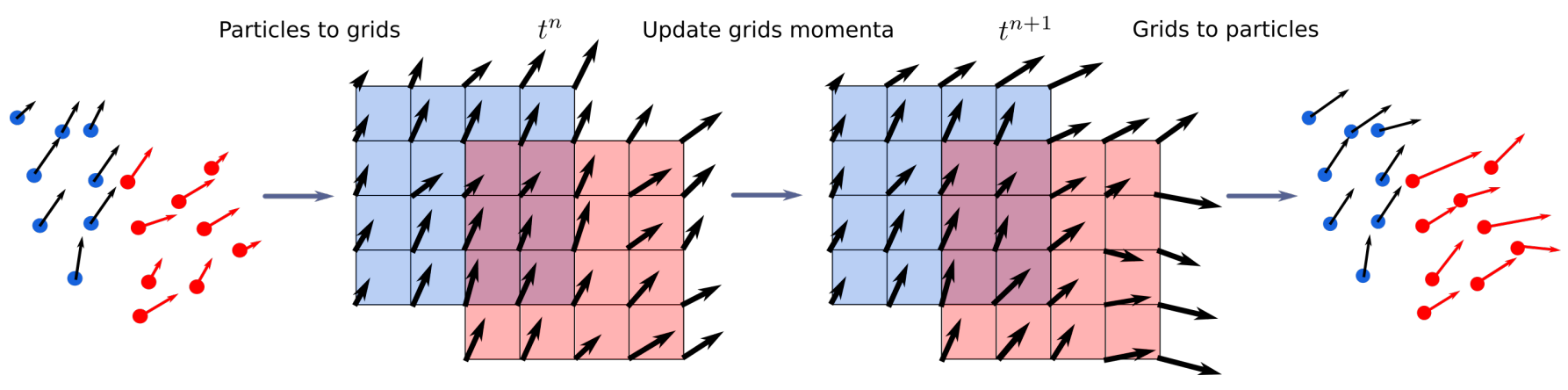

Fig. 7. MPM algorithm with two grids. We use separate water and sand grids. The blue and red dots denote water and sand particles respectively. In the overlapping region, we compute the momentum exchange term between the two species. We also compute the force based on individual constitutive model.

(2) Update grids momenta: Solving for the coupled water and sand grid velocities using semi-implicit backward Euler $(\S 4.2)$.

(3) Update particles: Update all particle state, including the cohesion based on saturation as well as plasticity return mappings (§4.3).

\subsection{Transfer to grid}

We transfer mass and momentum from sand and water particles to their respective grid using APIC [Jiang et al. 2015]. For each species $\alpha$, the particle $p$ interacts with the grid node ${ }_{i}^{\alpha}$ with weight $w_{i p}^{\alpha, n}=N\left(\mathbf{x}_{p}^{\alpha, n}-\mathbf{x}_{i}^{\alpha}\right)$. The weight is computed using the quadratic $\mathrm{B}$-spline interpolation kernel. Mass is computed according to

$$
m_{i}^{\alpha, n}=\sum_{p} w_{i p}^{\alpha, n} m_{p}^{\alpha}
$$

and velocity according to

$$
\mathbf{v}_{i}^{\alpha, n}=\frac{1}{m_{i}^{\alpha, n}} \sum_{p} w_{i p}^{\alpha, n} m_{p}^{\alpha}\left(\mathbf{v}_{p}^{\alpha, n}+\mathrm{C}_{p}^{\alpha, n}\left(\mathbf{x}_{i}^{\alpha, n}-\mathrm{x}_{p}^{\alpha, n}\right)\right),
$$

where the matrix $\mathrm{C}_{p}^{\alpha, n}$ is an extra matrix stored per particle which defines an affine velocity field local to the particle [Jiang et al. 2015] $\mathrm{C}_{p}^{\alpha, n}$ is initialized with $\mathrm{C}_{p}^{\alpha, 0}=0$ and updated at the end of the previous time step during the grid-to-particle transfer (Equation 29).

\subsection{Update Grids Momenta}

Using MPM, the forces in the sand and water phases are computed as

$$
\begin{aligned}
\mathbf{f}_{i}^{s}\left(\hat{\mathbf{x}}^{s}\right) & =-\frac{\partial \psi^{s}}{\partial \hat{\mathbf{x}}_{i}^{s}}=-\sum_{p} V_{p}^{0}\left(\frac{\partial \psi^{U}}{\partial \mathbf{F}^{s}}\left(\mathbf{F}_{p}^{s E}\left(\hat{\mathbf{x}}^{s}\right)\right)\left(\mathbf{F}_{p}^{s E, n}\right)^{\top} \nabla w_{i p}^{s, n},\right. \\
\mathbf{f}_{i}^{w}\left(\hat{\mathbf{x}}^{w}\right) & =-\frac{\partial \psi^{w}}{\partial \hat{\mathbf{x}}_{i}^{w}}=-\sum_{p} V_{p}^{0}\left(\frac{\partial \psi^{w}}{\partial J^{w}}\left(J^{w}\left(\hat{\mathbf{x}}^{w}\right)\right)\right) J^{w, n} \nabla w_{i p}^{w, n} .
\end{aligned}
$$

As in [Stomakhin et al. 2013] we think of $\hat{\mathbf{x}}_{i}^{\alpha}$ as the position of the grid node $i$ corresponding to species $\alpha$ that has been deformed from its original position $\mathrm{x}_{i}^{\alpha}$ by an amount of $\Delta t \mathrm{v}_{i}^{\alpha, n+1}$, i.e. $\hat{\mathbf{x}}_{i}^{\alpha}=$ $\hat{\mathbf{x}}_{i}^{\alpha}\left(\mathbf{v}_{i}^{\alpha, n+1}\right)=\mathbf{x}_{i}^{\alpha, n}+\Delta t \mathbf{v}_{i}^{\alpha, n+1}$. The discrete momentum balance to be solved is

$$
\begin{aligned}
m_{i}^{s, n}\left(\mathbf{v}_{i}^{s, n+1}-\mathbf{v}_{i}^{s, n}\right) & =\Delta t\left(\mathbf{f}_{i}^{s}\left(\hat{\mathbf{x}}^{s}\right)+m_{i}^{s, n} \mathbf{g}+\mathbf{d}_{i}^{s}(\hat{\mathbf{x}})\right) \\
m_{j}^{w, n}\left(\mathbf{v}_{j}^{w, n+1}-\mathbf{v}_{j}^{w, n}\right) & =\Delta t\left(\mathbf{f}_{j}^{s}\left(\hat{\mathbf{x}}^{w}\right)+m_{j}^{w, n} \mathbf{g}+\mathbf{d}_{j}^{w}(\hat{\mathbf{x}})\right),
\end{aligned}
$$

where the discrete interaction term is given by

$$
\begin{aligned}
& \mathbf{d}_{i j}^{s}(\hat{\mathbf{x}})=-c_{E} m_{i}^{s} m_{j}^{w}\left(\mathbf{v}_{i}^{s, n+1}-\mathbf{v}_{j}^{w, n+1}\right), \\
& \mathbf{d}_{j i}^{w}(\hat{\mathbf{x}})=c_{E} m_{i}^{s} m_{j}^{w}\left(\mathbf{v}_{i}^{s, n+1}-\mathbf{v}_{j}^{w, n+1}\right),
\end{aligned}
$$

for some drag coefficient $c_{E}$. Setting

$$
\mathbf{M}=\left(\begin{array}{cc}
\mathbf{M}^{s, n} & \\
& \mathbf{M}^{w, n}
\end{array}\right), \mathbf{v}=\left(\begin{array}{c}
\mathbf{v}^{s} \\
\mathbf{v}^{w}
\end{array}\right), \mathbf{f}\left(\hat{\mathbf{x}}\left(\mathbf{v}^{n+1}\right)\right)=\left(\begin{array}{c}
\mathbf{f}^{s}\left(\hat{\mathbf{x}}^{s}\right) \\
\mathbf{f}^{w}\left(\hat{\mathbf{x}}^{w}\right)
\end{array}\right),
$$

and $\mathbf{D}$ to be the drag coefficient matrix derived from Equations 20 and 21 , we can write the coupled system as

$$
(\mathbf{M}+\Delta t \mathbf{D}) \mathbf{v}^{n+1}=\mathbf{M v}^{n}+\Delta t\left(\mathbf{M g}+\mathbf{f}\left(\hat{\mathbf{x}}\left(\mathbf{v}^{n+1}\right)\right)\right) .
$$

At each time step, we solve this nonlinear system using a few iterations of a modified Newton's method. Since the matrix D is symmetric, and both $\mathbf{f}^{s}$ and $\mathbf{f}^{w}$ are derived as the negative gradient of a potential, the whole system is symmetric when linearized (assuming the effects of plasticity are ignored in the linearization) and can be solved using MINRES. We note that we do not include the effect of plasticity when computing the derivatives of $\mathrm{f}^{s}$. Doing otherwise results in non-symmetric sand force derivatives which would require GMRES. Our omission of these terms in the linearization of the system is a modification to the standard Newton's method. However, it is essential that we use implicit time stepping because of the stiff momentum exchange terms and our lagged plasticity approach is the key to making this efficient. Notably, this can lead to cohesion artifacts without the unilateral modification in (§3.1.1) (see Figure 10).

\subsection{Update Particles}

4.3.1 Update $J^{w}$. We do not keep track of the deformation gradient $\mathbf{F}^{w}$ of water particles, instead we keep track of its determinant $J^{w}$, which is updated according to the discretization of Equation 12, i.e.

$$
J_{p}^{w, n+1}=\left(\mathbf{I}+\Delta t \operatorname{tr}\left(\nabla \mathbf{v}_{p}^{w, n+1}\right)\right) J^{w, n}
$$



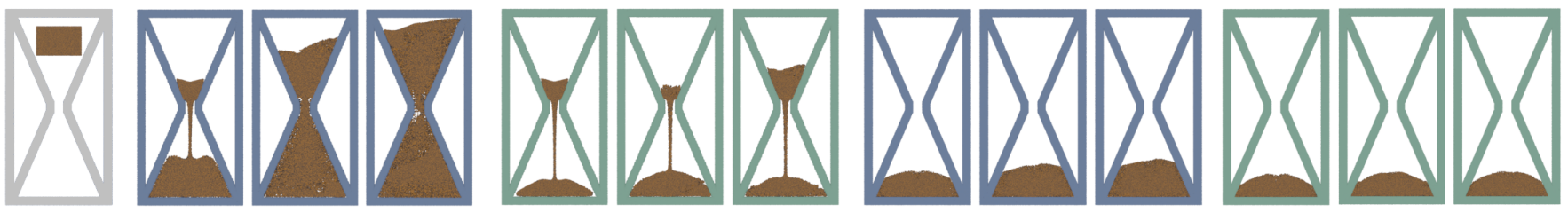

Fig. 8. 2D hourglass. An hourglass is flipped two times. The left most hourglass shaded grey depicts the initial state of sand at the beginning of the simulation. Each set-of-three figures depicts the state of the sand after going through the neck the first time, and after flipping the hourglass for the first and second time. The first two sets and the last two sets have grid $d x=7.4 \times 10^{-3}$ and $d x=3.7 \times 10^{-3}$ respectively. The first and third set are run without any volume correction fix, while the second and last set are run with our volume fix.

We found that in practice, this discretization tends to offer more stability than the alternative of evolving $\mathbf{F}^{w}$ followed by computing its determinant.

4.3.2 Update $\mathrm{F}^{s}$. Because we ignore the effects of plasticity during the implicit solve for momenta, $\hat{\mathbf{F}}^{s E, n+1}$ evolves with the grid during the grid momentum update as in Stomakhin et al. [2013]

$$
\hat{\mathbf{F}}_{p}^{s E, n+1}=\left(\mathbf{I}+\Delta t \nabla \mathbf{v}_{p}^{s, n+1}\right) \mathbf{F}^{s E, n} .
$$

$\hat{\mathbf{F}}_{p}^{s E, n+1}$ is later processed for plasticity at the end of the time step to define $\mathrm{F}_{p}^{s E, n+1}$, which we discuss below.

4.3.3 Saturation based cohesion. We define the water saturation on sand particles based on a heuristic. First, we populate a grid whose domain is the union of the sand grid and water grid domain. We mark each node that has a non-zero mass for both sand and water species as $\phi_{i}^{w, n+1}=1$, otherwise $\phi_{i}^{w, n+1}=0$. One can think of this grid as tracking an indicator function of the overlap region between the sand and water constituents. We then compute the saturation on the sand particle by interpolating from the grid to the sand particle according to

$$
\phi_{p}^{s, n+1}=\sum_{i} w_{s, i p}^{n} \phi_{i}^{w, n+1}
$$

This approximates the saturation as equal to one (maximal) deep in the interior of the overlap region with a ramp to zero exterior to the region. In all of our multi-species examples, we assume that the wet sand is already saturated and has reached its maximum cohesion level. Hence, any amount of additional water saturation will lower the cohesion level of the sand. We vary cohesion in a linear fashion as a function of water saturation as discussed in Section 3.4

$$
c_{C p}^{s, n+1}=c_{C p}^{s, 0}\left(1-\phi_{p}^{w, n+1}\right)
$$

where we note that the saturation is always in $(0,1)$. The approximation of the saturation in Equation 24 has errors biased towards full saturation in the interior. This naturally leads to more rapid failure in the landslides and debris flows we consider in our examples since the cohesion decreases more rapidly than it should. This is an extreme simplification to correct behavior defined in Robert and Soga [2013], but we found that it was effective for simulating these phenomena.

4.3.4 Projection and volume correction treatment. We now describe the plastic projection of $\hat{\mathrm{F}}^{s E, n+1}$ to $\mathrm{F}^{s E, n+1}$. We start from the Drucker-Prager projection, $P$, as implemented by Klár et al. [2016].
$P$ projects strains outside the yield surface onto the yield surface according to the plastic flow rule. The yield region has the shape of a cone. When the material is under expansion, the strain is projected to the tip. Otherwise it is projected to the side of the cone. However, while this approach is adequate for projection directions perpendicular to the hydrostatic axis, steps which project to the tip can induce volume gain. This occurs when a particle undergoes expansion that induces a cohesive elastic stress. In this case, stress is projected to the tip, which is a stress free state. The particle is then in a new rest state and any motion that would return it to its initial volume would be penalized elastically. This phenomena can lead to some clearly non-physical behavior (see Figure 8) and there are existing corrections to this in the mechanics literature, e.g. Dunatunga and Kamrin [2015] model material as a disconnected stress-free medium under sufficient expansion.

We combat this artifact by giving each sand particle an extra scalar attribute $v_{c p}^{s}$ which tracks changes in the log of the volume gained during extension. This can be naturally taken into account in the logarithmic-strain-based constitutive model to allow for compression in the event of prior net expansion. At each time step, we update $v_{c p}^{s}$ according to

$$
v_{c p}^{s, n+1}=v_{c p}^{s, n}+\log \left(\operatorname{det}\left(\mathbf{F}^{s E, n+1}\right)\right)-\log \left(\operatorname{det}\left(\hat{\mathbf{F}}^{s E, n+1}\right)\right),
$$

where $v_{c p}^{s, 0}=0$. Finally, when we perform the Drucker-Prager projection, we update $\epsilon^{s E, n+1}$ according to

$$
\boldsymbol{\epsilon}^{s E, n+1}=P\left(\hat{\boldsymbol{\epsilon}}^{s E, n+1}+\frac{v_{c p}^{n}}{d} \mathbf{I}\right),
$$

where $P$ is the projection operator described above.

This can be interpreted as projecting the elastic strain plus the volume gain term. Lastly, $\mathbf{F}^{s E, n+1}=\mathbf{U} \hat{\boldsymbol{\epsilon}}^{s E, n+1} \mathbf{V}^{\top}$ where $\hat{\mathbf{F}}^{s E, n+1}=$ $\mathrm{U} e^{\hat{\Sigma}^{s E, n+1}} \mathbf{V}^{\top}$ and $\hat{\boldsymbol{\epsilon}}^{s E, n+1}=\log \left(\hat{\Sigma}^{s E, n+1}\right)$. See Figures 8 and 9 for a demonstration of this effect. We note that this projection is particularly defined for constitutive models written in terms of the logarithmic strain. For a more general constitutive model, it would require non-trivial modification.

4.3.5 Update position and velocity. Velocity is updated according to

$$
\mathbf{v}_{p}^{\alpha, n+1}=\sum_{i} w_{i p}^{\alpha, n} \mathbf{v}_{i}^{\alpha, n+1}
$$




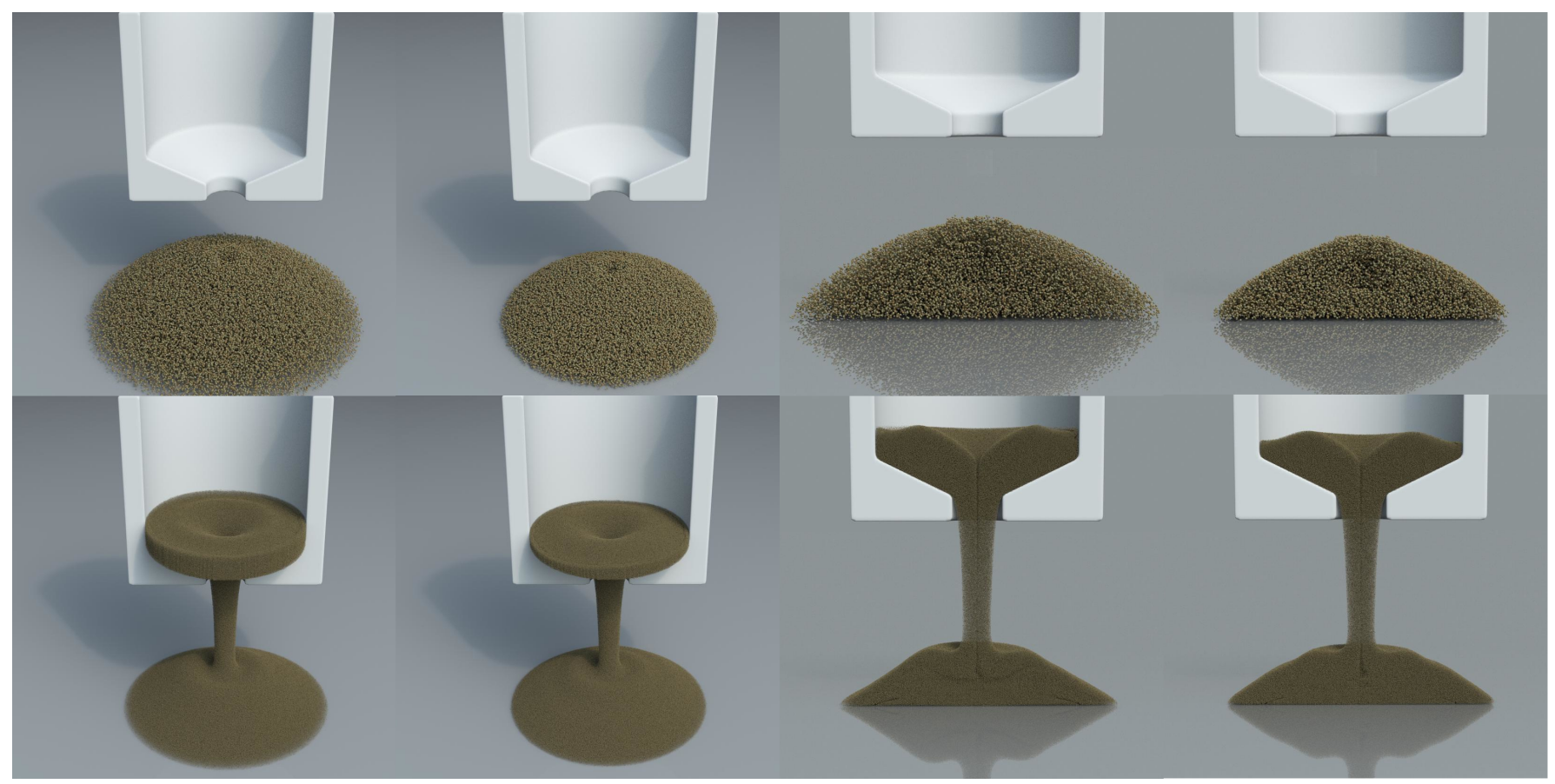

Fig. 9. Pouring sand. The top row depicts the result of an explicit simulation with a coarser grid size $\left(d x=10^{-2}\right)$, while the bottom row corresponds to a finer grid size $\left(d x=10^{-3}\right)$. The first and third column correspond to a projection step without volume correction, while the second and fourth column uses our volume correction algorithm.

the affine velocity matrix is updated according to

$$
\mathrm{C}_{p}^{\alpha, n+1}=\sum_{i} w_{i p}^{n} \mathbf{v}_{i}^{\alpha, n+1}\left(\left(\frac{4}{h^{2}}\right)\left(\mathbf{x}_{i}^{\alpha, n}-\mathbf{x}_{p}^{\alpha, n}\right)\right)^{\top},
$$

where $h$ is the Eulerian grid spacing. Lastly position is updated according to

$$
\mathrm{x}_{p}^{\alpha, n+1}=\mathrm{x}_{p}^{\alpha, n}+\Delta t \mathrm{v}_{p}^{\alpha, n+1}
$$

\section{IMPLEMENTATION AND RESULTS}

We use the sparse grid structure provided by OpenVDB [Museth 2013]. The table of performance and parameter for various examples are listed in Table 2.

\subsection{Unilateral hyperelasticity and implicit time stepping}

Figure 10 demonstrates the effects of our unilateral constitutive model with semi-implicit time stepping. We note that our unilateral potential removes artificial cohesion effects in the simulation of dry sand. The cohesion in this simulation is zero so the sand should not stick together. When we use the constitutive model from [Klár et al. 2016] with a semi-implicit time integration scheme, we see artificial cohesion that gets worse as we increase the time step size. Using our unilateral elastic energy function removes this artificial cohesion. We further demonstrate that our semi-implicit scheme gives results comparable to the more accurate, but more expensive fully implicit backward Euler scheme in Figure 11. The importance of choosing the right unilateral parameters is illustrated by Figure 6 .
If the regions $A$ and $B$ in Figure 5 do not closely fit the DruckerPrager cone, then the accuracy of the simulation is compromised for large time steps.

\subsection{Volume fix and wet sand}

To illustrate our volume fix to the Drucker-Prager artifact, we run a simulation of an hourglass turned three times with explicit symplectic Euler, as shown by Figure 8 . The left most figure depicts the initial state of the sand. For each set of three hourglass figures, we show the state of the sand after going through the neck the first time, after the first flip, and after the second flip. Figure 9 depicts a similar artifact in a 3D piling of sand. Without the volume correction algorithm and without a sufficiently small grid resolution, we observe a substantial volume gain artifact.

\subsection{Wet sand and dam breach}

In Figure 3 we demonstrate how varying cohesion gives rise to different wet sand behaviors. In Figure 2 we demonstrate our approach with an example that is representative of the types of gravity driven flows we are interested in with our approach. As water flows into the wall of a dam, the saturation increases weakening it. The cohesion of sand decreases with saturation and the dam eventually breaks.

We demonstrate the effect of the active component of the momentum exchange terms in Equation 13 using a simulation of a 2D dam breach, shown in Figure 4. Again water pours in from a reservoir and slowly erodes a retaining wall. We note that the active term has only a subtle effect on the bulk dynamics of the motion for these 

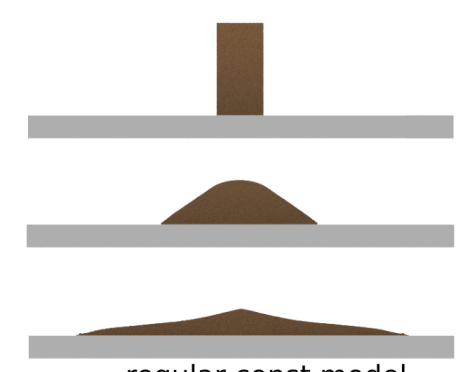

regular const model explicit max_dt $=1 \mathrm{e}-4$
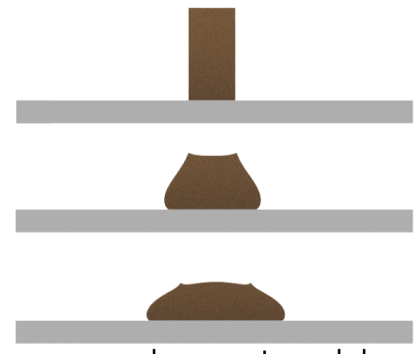

regular const model semi-implicit max_dt $=1 \mathrm{e}-3$

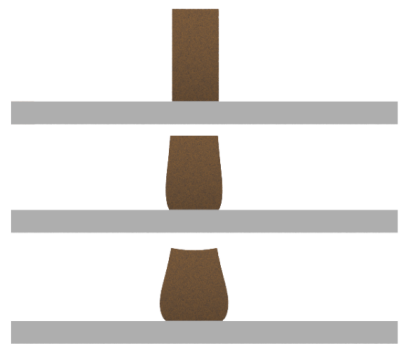

regular const model semi-implicit max_dt $=1 \mathrm{e}-2$

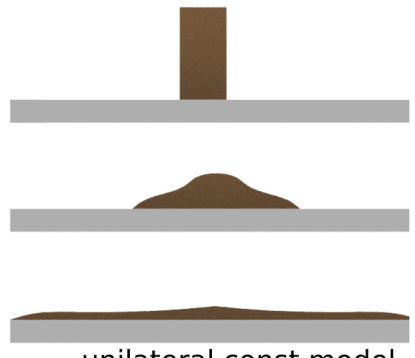

unilateral const model semi-implicit max_dt $=1 \mathrm{e}-2$

Fig. 10. 2D sand pile. From top to bottom row: initial, middle, and final configuration of a 2D sand pile. The left column is an explicit simulation with maximum $\Delta t=10^{-4}$. The two columns in the middle are the results of semi-implicit simulation with the regular constitutive model and max $\Delta t=10^{-2}$ and $10^{-3}$ respectively. Note that both suffer from artificial cohesion, although it diminishes with smaller $\Delta t$. On the right is semi-implicit time stepping with our unilateral energy density function with maximum $\Delta t=10^{-2}$. Note that it does not suffer from artificial cohesion.

types of flows. We discretize the active term by adding

$$
\sum-p_{p}^{w, n} \nabla w_{i p}^{s, n} \frac{m_{i}^{w, n}}{m_{i}^{s, n}+m_{i}^{w, n}}
$$

to the water drag term in Equation 21. We then define the solid drag term to be equal and opposite to the water drag in accordance with the zero-net-sum nature of the momentum exchange.

\section{LIMITATIONS AND FUTURE WORK}

Our approach has a number of limitations. The momentum exchange model we use in the water/sand multispecies examples is rather simplified. While adequate for gravity driven flows like landslides and levee breaches, it is inadequate for capillary driven phenomena like water being drawn in to dry sand. Such phenomena has been captured by prior approaches like that of Lenaerts and Dutre [2009]. Furthermore we fail to capture behavior like those in Rungjiratananon et al. [2008] where surface tension effects in wetting are more accurately captured.

Although our approximation to the dependence of sand cohesion on saturation is useful for facilitating rapid failure of water/sand mixtures, it is an extreme simplification to the correct behavior defined in Robert and Soga [2013] and this compromises its accuracy dramatically. This reduces the applicability of our approach outside of visually plausible simulation applications.

Large values of the momentum exchange coefficient $c_{E}$ can lead to ill-conditioning in the linear systems that arise during implicit time stepping. We found that these cases required many MINRES iterations to resolve and lead to excessive run times. This complicated the simulation of slurry materials where the water and sand remain mixed. In the future we would like to examine appropriate preconditioners to improve the performance. Also, while we omit or use a very simplistic buoyancy term for the reversible momenta exchange in the $\mathbf{p}^{\alpha}$ equations, we would like to examine the addition of more accurate terms to produce phenomena like absorbent sponges interacting with liquids. Lastly, we would like to examine the suitability of our multiple grid MPM framework for the simulation of more general multi-species interactions like chemically reacting flow.

\section{ACKNOWLEDGMENTS}

The authors thank Qi Guo, Danielle Joseph, Lawrence Lee, and Stephanie Wang for their help with simulations, rendering, and video production. The work is supported by NSF CCF-1422795, ONR (N000141110719, N000141210834), DOD (W81XWH-15-1-0147), Intel STC-Visual Computing Grant (20112360).

\section{REFERENCES}

K. Abe, K. Soga, and S. Bandara. 2014. Material Point Method for Coupled Hydromechanical Problems. F Geotech Geoenv Eng 140, 3 (2014), 04013033.

I. Alduán and M. Otaduy. 2011. SPH granular flow with friction and cohesion. In Proc ACM SIGGRAPH/Eurograph Symp Comp Anim. 25-32.

R. Atkin and R. Craine. 1976. Continuum theories of mixtures: basic theory and historical development. Quart 7 Mech App Math 29, 2 (1976), 209-244.

S. Bandara, A. Ferrari, and L. Laloui. 2016. Modelling landslides in unsaturated slopes subjected to rainfall infiltration using material point method. Int 7 Num Anal Meth Geomech 40, 9 (2016), 1358-1380.

S. Bandara and K. Soga. 2015. Coupling of soil deformation and pore fluid flow using material point method. Comp Geotech 63 (2015), 199-214.

K. Bao, X. Wu, H. Zhang, and E. Wu. 2010. Volume fraction based miscible and immiscible fluid animation. Comp Anim Virtual Worlds 21, 3-4 (2010), 401-410.

M. Becker and M. Teschner. 2007. Weakly compressible SPH for free surface flows. In ACM SIGGRAPH/Eurograph Symp Comp Anim, D. Metaxas and J. Popovic (Eds.). The Eurographics Association.

J. Bonet and R. Wood. 2008. Nonlinear continuum mechanics for finite element analysis. Cambridge University Press.

R. Borja. 2006. On the mechanical energy and effective stress in saturated and unsaturated porous continua. Int 7 Solids Struct 43 (2006), 1764-1786.

G. Daviet and F. Bertails-Descoubes. 2016. A Semi-implicit Material Point Method for the Continuum Simulation of Granular Materials. ACM Trans Graph 35, 4 (2016), 102:1-102:13.

D. Drucker and W. Prager. 1952. Soil mechanics and plasticity analysis or limit design. Quart App Math 10 (1952), 157-165.

D. Drumheller. 2000. On theories for reacting immiscible mixtures. Int f Eng Sci 38, 3 (2000), 347 - 382 .

S. Dunatunga and K. Kamrin. 2015. Continuum modelling and simulation of granular flows through their many phases. F Fluid Mech 779 (2015), 483-513.

X. He, H. Wang, F. Zhang, H. Wang, G. Wang, K. Zhou, and E. Wu. 2015. Simulation of Fluid Mixing with Interface Control. In Proc ACM SIGGRAPH / Eurograph Symp Comp Anim. ACM, 129-135.

M. Ihmsen, A. Wahl, and M. Teschner. 2013. A Lagrangian framework for simulating granular material with high detail. Comp Graph 37, 7 (2013), 800-808.

I. Jassim, D. Stolle, and P. Vermeer. 2013. Two-phase dynamic analysis by material point method. Int 7 Num Anal Meth Geomech 37 (2013), 2502-2522.

C. Jiang, C. Schroeder, A. Selle, J. Teran, and A. Stomakhin. 2015. The Affine ParticleIn-Cell Method. ACM Trans Graph 34, 4 (2015), 51:1-51:10.

N. Kang, J. Park, J. Noh, and S. Shin. 2010. A Hybrid Approach to Multiple Fluid Simulation using Volume Fractions. Comp Graph Forum 29, 2 (2010), 685-694. 


\begin{tabular}{ccccccccc}
\hline Example & sec/frame & Scheme & $\max \Delta t$ & Particle \# & $\Delta x$ & $\rho^{s} / E / v$ & $a / b / c_{0}$ & $\rho^{w} / k / \gamma$ \\
\hline 2D Hourglass (Fig. 8) & 0.84 & Explicit & $5 \times 10^{-4}$ & $3.0 \times 10^{4}$ & $3.7 \times 10^{-3}$ & $0.2 / 5 / 0.4$ & - & - \\
2D Hourglass (Fig. 8) & 0.73 & Explicit & $5 \times 10^{-4}$ & $3.0 \times 10^{4}$ & $7.4 \times 10^{-3}$ & $0.2 / 5 / 0.4$ & - & - \\
2D Piling (Fig. 10) & 7.26 & Explicit & $5 \times 10^{-5}$ & $4.7 \times 10^{4}$ & $2.5 \times 10^{-3}$ & $20 / 100 / 0.4$ & - & - \\
2D Piling (Fig. 10) & 3.39 & Semi-Implicit & $1 \times 10^{-4}$ & $4.7 \times 10^{4}$ & $2.5 \times 10^{-3}$ & $20 / 100 / 0.4$ & - & - \\
2D Piling Unilateral (Fig. 10) & 0.67 & Semi-Implicit & $1 \times 10^{-2}$ & $4.7 \times 10^{4}$ & $2.5 \times 10^{-3}$ & $20 / 100 / 0.4$ & $-3.0 / 0 / 1 \times 10^{-2}$ & - \\
Implicit Piling Unilateral (Fig. 11) & 24.30 & Semi-Implicit & $1 \times 10^{-3}$ & $9.4 \times 10^{3}$ & $4 \times 10^{-3}$ & $2 / 100 / 0.4$ & $-3 \times 10^{-3} / 0 / 1 \times 10^{-2}$ & - \\
Dry Sand Wedge (Fig. 3) & 129.65 & Explicit & $1 \times 10^{-4}$ & $1.0 \times 10^{6}$ & $3.0 \times 10^{-3}$ & $4 / 19 / 0.4$ & - & - \\
Wet Sand Wedge (Fig. 3) & 72.65 & Semi-Implicit & $1 \times 10^{-3}$ & $1.0 \times 10^{6}$ & $3.0 \times 10^{-3}$ & $4 / 24 / 0.4$ & - & - \\
2D Dam Breach (Fig. 4) & 59.09 & Semi-Implicit & $1 \times 10^{-3}$ & $1.7 \times 10^{5}$ & $1.15 \times 10^{-2}$ & $2 / 400 / 0.4$ & - & $2 / 50 / 3$ \\
3D Dam Breach (Fig. 2) & 268.99 & Semi-Implicit & $1 \times 10^{-3}$ & $3.0 \times 10^{6}$ & $1.0 \times 10^{-2}$ & $2 / 100 / 0.4$ & $-0.1 / 100 c_{C} / 1 \times 10^{-2}$ & $2 / 100 / 7$ \\
\hline
\end{tabular}

Table 2. Table of performance and parameters.
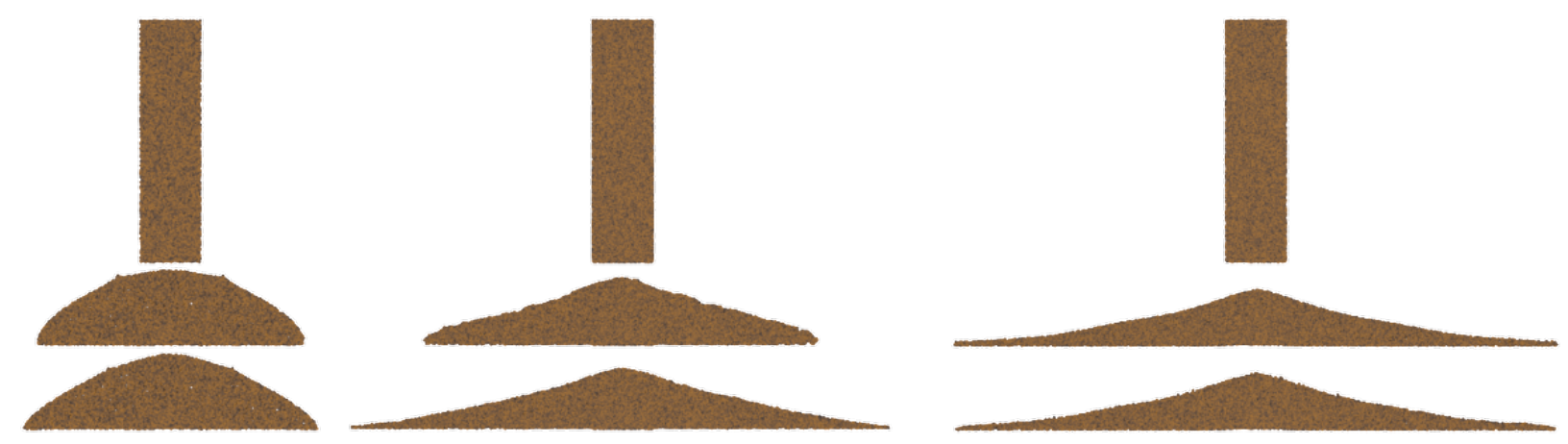

Fig. 11. Fully implicit unilateral. Comparison of increasingly implicit time stepping schemes for 2D sand column collapse. The simulations on the left and at the center are run with the semi-implicit scheme with the regular and unilateral energy density functions respectively. The right-most figure shows a fully implicit scheme with our modified energy density function. Note that the unilateral density function with semi-implicit time stepping yields a good approximation of the fully implicit result, while the regular energy density suffers from artificial cohesion.

G. Klár, T. Gast, A. Pradhana, C. Fu, C. Schroeder, C. Jiang, and J. Teran. 2016. Druckerprager Elastoplasticity for Sand Animation. ACM Trans Graph 35, 4 (2016), 103:1103:12.

T. Lenaerts and P. Dutré. 2009. Mixing Fluids and Granular Materials. Comp Graph Forum 28, 2 (2009), 213-218.

S. Liu, Z. Wang, Z. Gong, and Q. Peng. 2008. Simulation of Atmospheric Binary Mixtures Based on Two-fluid Model. Graph Mod 70, 6 (2008), 117-124.

F. Losasso, J. Talton, N. Kwatra, and R. Fedkiw. 2008. Two-Way Coupled SPH and Particle Level Set Fluid Simulation. IEEE Trans Visu Comp Graph 14, 4 (2008), 797-804.

P. Mackenzie-Helnwein, P. Arduino, W. Shin, J. Moore, and G. Miller. 2010. Modeling strategies for multiphase drag interactions using the material point method. Int $\mathcal{F}$ Num Meth Eng 83, 3 (2010), 295-322.

C. Mast, P. Arduino, G. Miller, and M. Peter. 2014. Avalanche and landslide simulation using the material point method: flow dynamics and force interaction with structures. Comp Geosci 18, 5 (2014), 817-830.

V. Mihalef, D. Metaxas, and M. Sussman. 2009. Simulation of two-phase flow with sub-scale droplet and bubble effects. Comp GraphForum 28, 2 (2009), 229-238.

Ken Museth. 2013. VDB: High-resolution Sparse Volumes with Dynamic Topology. ACM Trans. Graph. 32, 3, Article 27 (July 2013), 22 pages.

R. Narain, A. Golas, and M. Lin. 2010. Free-flowing granular materials with two-way solid coupling. ACM Trans Graph 29, 6 (2010), 173:1-173:10.

M. Nielsen and O. Osterby. 2013. A Two-continua Approach to Eulerian Simulation of Water Spray. ACM Trans Graph 32, 4 (2013), 67:1-67:10.

D. Peachey. 1986. Modeling Waves and Surf. SIGGRAPH Comput Graph 20, 4 (1986), 65-74.

D. Ram, T. Gast, C. Jiang, C. Schroeder, A. Stomakhin, J. Teran, and P. Kavehpour. 2015. A material point method for viscoelastic fluids, foams and sponges. In Proc ACM SIGGRAPH/Eurograph Symp Comp Anim. 157-163.

B. Ren, Y. Jiang, C. Li, and M. Lin. 2015. A simple approach for bubble modelling from multiphase fluid simulation. Comp Vis Media 1, 2 (2015), 171-181.

B. Ren, C. Li, X. Yan, M. Lin, J. Bonet, and S. Hu. 2014. Multiple-Fluid SPH Simulation Using a Mixture Model. ACM Trans Graph 33, 5 (2014), 171:1-171:11.

D. Robert and K. Soga. 2013. Soil-Pipeline Interaction in Unsaturated Soils. In Mechanics of Unsaturated Geomaterials, Lyesse Laloui (Ed.). Wiley Online Library, Chapter 13, 303-325.

W. Rungjiratananon, Z. Szego, Y. Kanamori, and T. Nishita. 2008. Real-time Animation of Sand-Water Interaction. Comp Graph Forum 27, 7 (2008), 1887-1893.
O. Song, H. Shin, and H. Ko. 2005. Stable but Nondissipative Water. ACM Trans Graph 24, 1 (2005), 81-97.

A. Stomakhin, C. Schroeder, L. Chai, J. Teran, and A. Selle. 2013. A Material Point Method for snow simulation. ACM Trans Graph 32, 4 (2013), 102:1-102:10.

A. Stomakhin, C. Schroeder, C. Jiang, L. Chai, J. Teran, and A. Selle. 2014. Augmented MPM for phase-change and varied materials. ACM Trans Graph 33, 4 (2014), 138:1138:11.

D. Sulsky, S. Zhou, and H. Schreyer. 1995. Application of a particle-in-cell method to solid mechanics. Comp Phys Comm 87, 1 (1995), 236-252.

T. Takahashi, H. Fujii, A. Kunimatsu, K. Hiwada, T. Saito, K. Tanaka, and H. Ueki. 2003. Realistic Animation of Fluid with Splash and Foam. Comp Graph Forum 22, 3 (2003), 391-400.

N. Thürey, F. Sadlo, S. Schirm, M. Müller-Fischer, and M. Gross. 2007. Real-time Simulations of Bubbles and Foam Within a Shallow Water Framework. In Proc ACM SIGGRAPH/Eurograph Symp Comp Anim. Eurographics Association, 191-198.

L. Yang, S. Li, A. Hao, and H. Qin. 2014. Hybrid Particle-grid Modeling for Multi-scale Droplet/Spray Simulation. Comp Graph Forum 33, 7 (2014), 199-208.

T. Yang, J. Chang, B. Ren, M. Lin, J. Zhang, and S. Hu. 2015. Fast Multiple-fluid Simulation Using Helmholtz Free Energy. ACM Trans Graph 34, 6 (2015), 201:1-201:11.

Y. Yue, B. Smith, C. Batty, C. Zheng, and E. Grinspun. 2015. Continuum foam: a material point method for shear-dependent flows. ACM Trans Graph 34, 5 (2015), 160:1-160:20.

Y. Zhu and R. Bridson. 2005. Animating sand as a fluid. ACM Trans Graph 24, 3 (2005), 965-972. 